\title{
On the fundamental role of oxygen for the photochromic effect of $\mathrm{WO}_{3}$
}

\author{
C. Bechinger, G. Oefinger, S. Herminghaus, and P. Leiderer \\ Fakultät für Physik, Universität Konstanz, D-78434 Konstanz, Germany
}

(Received 26 February 1993; accepted for publication 11 June 1993)

The photochromic effect of thin amorphous $\mathrm{WO}_{3}$ films, i.e., the coloration upon exposing the sample to ultraviolet light, has been investigated by means of optically excited surface plasmons. Due to the high sensitivity of this technique, it was possible to investigate the dynamics of this effect on a scale as short as seconds. Our results demonstrate the dominant role of oxygen during the coloration and bleaching processes. We found clear evidence of an oxygen exchange between the sample and the ambient atmosphere during the photochromic process, which is at variance with the widely accepted double charge injection model. The coloration rate shows the same wavelength dependence as that of the creation rate of electron-hole pairs formed by optical excitation. The results can be explained by the light-induced decomposition of the incorporated water.

\section{INTRODUCTION}

Tungsten oxide is probably the most thoroughly investigated representative of a group of materials that are known as chromogenic. Their most distinct feature is that they can be continuously switched between two different optical states. This property is quite interesting, e.g., for "smart window" or display applications, and has been studied extensively. ${ }^{1,2}$ Thin amorphous $\mathrm{WO}_{3}$ films can be switched reversibly from transparent to a blue colored state which has a broad absorption band at $1000 \mathrm{~nm}$, with a shoulder towards the visible range. ${ }^{3}$ Coloration can be obtained, for example, by an electrochemical process (electrochromic effect) using an external voltage and a proton source, or by irradiation, e.g., with ultraviolet light (photochromic effect). To gain insight into the basic mechanisms taking place during the coloration, one has to take into account two conspicuous facts: first, the valency state of the tungsten atoms changes from $+V I$ to $+V^{4,5}$ and, second, a simultaneous structural change is observed and is ascribed to the formation of a hydrogen tungsten bronze $\left(\mathrm{H}_{x} \mathrm{WO}_{3}\right) .{ }^{6}$ Both features are usually discussed in terms of the double-charge-injection model (DCIM) $:^{7}$

$$
\mathrm{W}^{+V I} \mathrm{O}_{3}+x e^{-}+x \mathrm{H}^{+} \rightleftharpoons \mathrm{H}_{x} \mathrm{~W}_{x}^{+V} \mathrm{~W}_{1-x}^{+V I} \mathrm{O}_{3} \text {. }
$$

It implies that electrons and protons enter the $\mathrm{WO}_{3}$ film from the outside. This seems to be evident for the electrochromic process, where the sample is in contact with an electrolyte and an externally applied voltage which may act as a source for the required charge carriers. The electrons then create the color centers by reducing the valency of the tungsten atoms from $+V I$ to $+V$, as described by Eq. (1). The concentration of these color centers formed may be of the same order of magnitude as that of the tungsten atoms. Such a high concentration is attainable only when compensating charges like protons are present. This is also taken into consideration by Eq. (1), which describes the coloration and the bleaching of tungsten oxide by a simultaneous injection and an extraction, respectively, of electrons and protons.
Although this model is widely accepted, there exist some results which raise doubts on its validity. Rutherford backscatlering spectroscopy (RBS) measurements, for example, could not confirm the increased hydrogen content of electrochemically colored films predicted by the doublecharge-injection model. ${ }^{8}$ The latter is questioned further by a simple experiment: We found that electrochemically and photochemically colored samples can be bleached rapidly by oxidizing them with $\mathrm{H}_{2} \mathrm{O}_{2}$. Figure 1 (a) shows a 400nm-thin $\mathrm{WO}_{3}$ film after coloration with an indium wire and an $\mathrm{HCl}$ electrolyte. ${ }^{7}$ Figure 1 (b) demonstrates the result after a droplet of concentrated $\mathrm{H}_{2} \mathrm{O}_{2}$ has been deposited in the middle of the sample. In the area where the film was in contact with the strongly oxidizing fluid, total bleaching, which occurs within less than $1 \mathrm{~s}$, is observed. Afterwards the film can be colored again [Fig. 1(c)]. We repeated this procedure several times and noticed no change in coloration efficiency.

Although this result does not rule out the DCIM, it supports the idea that oxygen plays an important role during the bleaching process of tungsten oxide; up to now, however, there has been no direct experimental evidence for this. One reason might be the fact that most of the published work deals with the electrochromic effect; in this case also the electrolyte has to be considered and adds to the complexity of the investigated system. Furthermore, irreversible reactions and electrolysis that can even lead to degradation of the films can occur. ${ }^{9}$

Some of these complications could be avoided by using the photochromic effect, i.e., the light-induced change of the optical absorption of $\mathrm{WO}_{3}$. That there is, nevertheless, a lack of experimental data dealing with the photochromic coloration of tungsten oxide may be due to the comparatively small changes in the optical absorptivity of thin films upon irradiation as compared to the electrochromic process. Usually, light exposure times ranging from hours to days are required in order to observe differences in the transmission spectra. ${ }^{10}$ To overcome such long illumination times we used the attenuated total reflection (ATR) technique, which allows even very small changes in the optical constants of thin films to register, to assist the in- 

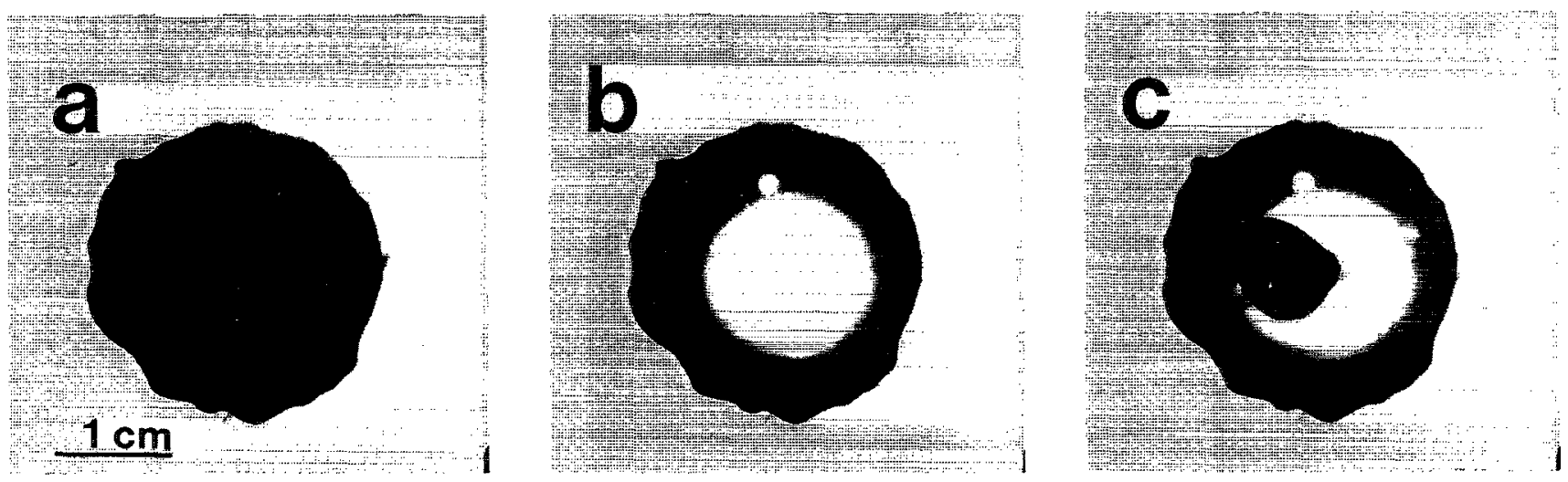

FIG. 1. Photographs of the reversible electrochromic effect of a 340-nm-thick $\mathrm{WO}_{3}$ film (a) after electrolytic coloration (approximate spot diameter 20 $\mathrm{mm}$ ), (b) after blcaching a part of the sample with $\mathrm{H}_{2} \mathrm{O}_{2}$, and (c) after coloration inside the area previously bleached.

vestigation of this work. In this way it was possible, for the first time, to observe the dynamics of the photochromic effect on a scale of seconds.

\section{EXPERIMENT}

Our samples were 10 - and 340 -nm-thick $\mathrm{WO}_{3}$ layers evaporated onto a silver film. Optically excited surface plasmons in the silver film were used to determine the real and the imaginary part of the dielectric function $\epsilon=\epsilon^{\prime}+i \epsilon^{\prime \prime}$. Here, we only want to describe the principle of the experimental setup; for details concerning the ATR method we refer to the work of Sambles et al. ${ }^{11}$ The light of a $p$-polarized HeNe laser is reflected from the base of a prism coated with a silver film and a thin tungsten oxide layer on top. The angle of incidence $\boldsymbol{\theta}$ of the light determines the light wave vector component $k_{\|}$parallel to the silver- $\mathrm{WO}_{3}$ boundary. Surface plasmons with a frequency of the laser light $\omega$ will be excited if their wave vector is matched by $k_{\|}$of the incident light. This gives rise to a dip in the reflected light intensity $I$ at $\Theta=\Theta_{R}$ where the resonance occurs. From the value of $\Theta_{R}$, information about the real part $\epsilon^{\prime}$ of the dielectric function is obtained. The depth of the minimum is most pronounced if radiation damping of the surface plasmons, caused by their decay into photons, equals the internal damping due to dissipation within the layer system. Changing the optical constants of tungsten oxide, and thus its electrical conductivity $\sigma(\omega)$, leads to a change in $I$. For a suitable thickness of the silver film one obtains a linear dependence between $\epsilon^{\prime \prime}$ in $\mathrm{WO}_{3}$ and the reflected light intensity in the resonance $I_{R}$. Since the refractive index $n$ of tungsten oxide varies only slightly during the coloration, ${ }^{12}$ a linear dependence between $\epsilon^{\prime \prime}$ and the extinction coefficient $\kappa$ is found. Thus changes in $I_{R}$ can be used as a direct measure of the change of the absorptivity of the $\mathrm{WO}_{3}$ films.

The investigated tungsten oxide layers were deposited on a 53-nm-thick silver film by thermal evaporation of $\mathrm{WO}_{3}$ pellets at a pressure of $10^{-5}$ mbar. During the evaporation process the substrate was at room temperature. $\mathrm{WO}_{3}$ films obtained in this way were amorphous, as probed by $x$-ray measurements. For irradiating the tungsten oxide layer a $150 \mathrm{~W}$ xenon high pressure lamp was used as a light source. Wavelengths $\lambda>450 \mathrm{~nm}$ were suppressed with filters in order to avoid thermal effects. The resulting UV intensities on the sample were determined with a power meter to be $350 \mathrm{~mW} / \mathrm{cm}^{2}$. In some experiments the spectral range of the incident radiation was restricted to a narrow band using a monochromator. There the intensities ranged from several nanowatts to about $200 \mu \mathrm{W} / \mathrm{cm}^{2}$ depending on the wavelength used.

\section{RESULTS}

An example of the effect of UV irradiation on a tungsten oxide layer is shown in Fig. 2. Both a shift of $\Theta_{R}$ to smaller angles and a simultaneous increase of the minimum of the reflectivity $R_{\text {res }}$ are observed. This corresponds to a decrease of $\epsilon_{1}$ and an increase of $\epsilon_{2}$, respectively,

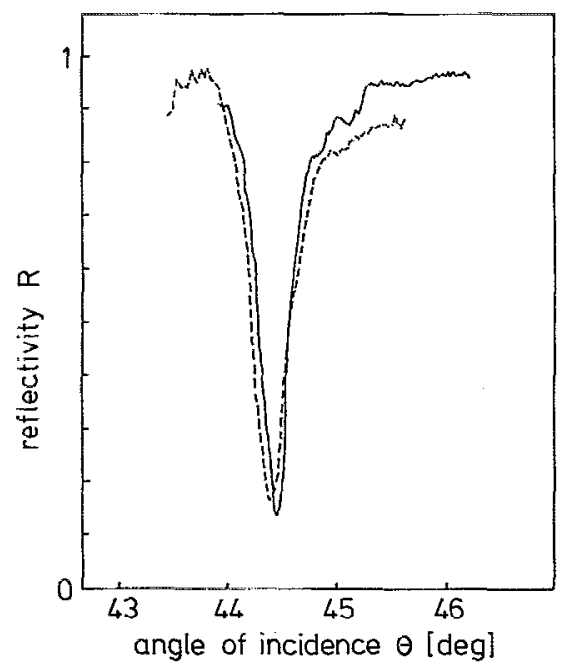

FIG. 2. Reflectivity $R$ as a function of the angle of incidence $\theta$ for a 10-nm-thick $\mathrm{WO}_{3}$ film before (solid line) and directly after (dashed line) 5 min UV irradiation. 


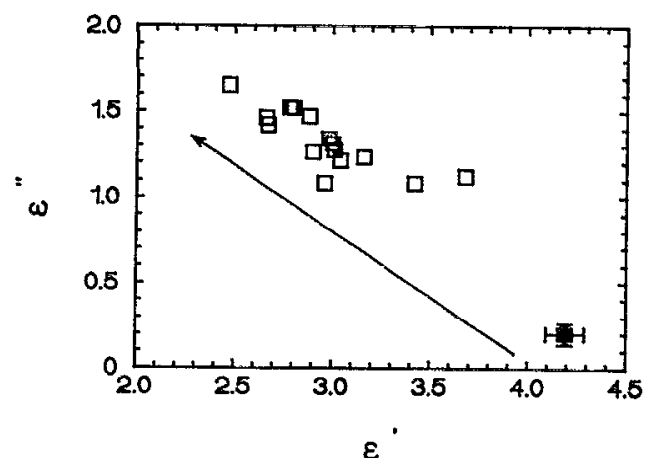

FIG. 3. Measured dependence of the imaginary and the real part of the dielectric function, $\epsilon^{\prime}$ and $\epsilon^{\prime \prime}$, for increasing absorption stages (the direction being indicated by the arrow) of 400 -nm-thick tungsten oxide samples. The filled square represents to a transparent sample. The data were taken using ellipsometry (after Ref. 12).

which is in agreement with the coloration behavior found by other authors using the electrochromic effect (Fig. 3). ${ }^{12}$

Next we will discuss the time dependence of the optical absorption of tungsten oxide caused by the photochromic process. Figure 4 shows a typical result of the photochromic behavior of a $10 \mathrm{~nm} \mathrm{WO}_{3}$ film. During exposure to ultraviolet light for $t_{e}=20 \mathrm{~s}$ in an oxygen atmosphere, layer absorption increases rapidly. Immediately after the irradiation is stopped by closing a shutter, the absorption decreases strongly and reaches its initial value after about $1000 \mathrm{~s}$.

\section{A. Bleaching process}

At first we want to concentrate on the bleaching process taking place after UV light has been turned off. In this range the absorption of the tungsten oxide is only determined by the dynamics of the decay of the color centers.

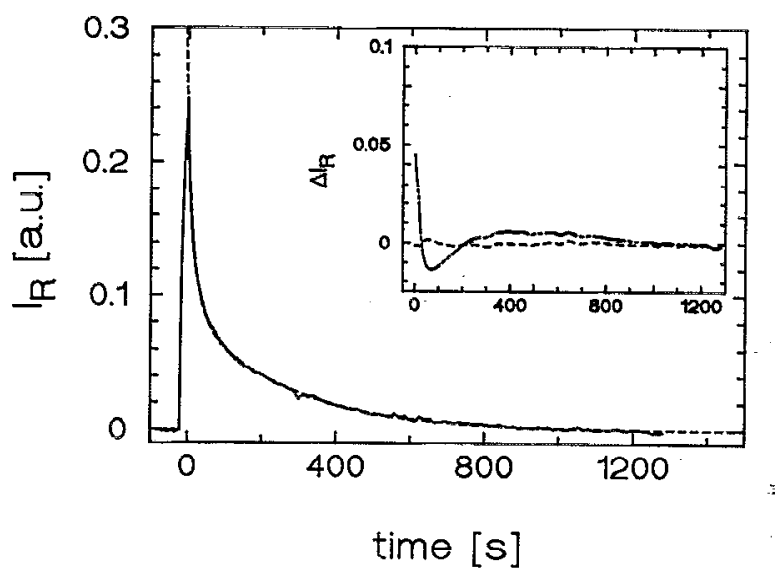

FIG. 4. Dynamics of reflected light intensity $I_{K}$, which is proportional to absorption, of a $10-\mathrm{nm}$-thick $\mathrm{WO}_{3}$ film during, and after $20 \mathrm{~s}$ of, UV irradiation in oxygen atmosphere. The dashed line corresponds to the sum of two exponentials [according to Eq. (2) and Table I]. The inset, at the same time scale, shows the deviation $\Delta I_{R}=\left(I_{R}^{\text {meas }}-I_{R}^{\text {itt }}\right)$ of the data from two fits: (i) the single exponential fit (dash-dotted line) and (ii) the sum of two exponentials (dashed line).
(In contrast, during UV irradiation the simultaneous processes of creation and decay of absorbing $W^{+V}$ states have to be taken into account.) Attempts to fit the bleaching behavior with an exponential decay, often present in relaxation processes, were not satisfying, as is evident from the inset of Fig. 4 (dashed-dotted line). Fitting instead a stretched exponential function ${ }^{13}$ to the data did not give considerable improvement. However, the sum of two exponentials

$$
I_{R}=A_{f} \exp \left(-t / \tau_{f}\right)+A_{s} \exp \left(-t / \tau_{s}\right)
$$

fits our data very well, as is shown in Fig. 4. In fact, the fit (dashed line) cannot be distinguished from the measured data within experimental accuracy. As Eq. (2) suggests, the bleaching process of tungsten oxide can be described by the sum of a fast and a slow process of two time constants $\tau_{f}$ and $\tau_{s}$, which dominate the behavior for the beginning and the end of the bleaching dynamics, respectively. For the case shown here, $\tau_{f}$ and $\tau_{s}$ differ by more than one order of magnitude, the corresponding amplitudes $A_{f}$ and $A_{s}$ differ by only about $25 \%$. Now we will present results for the dependence of these fit parameters on the experimental conditions.

\section{B. Exposure time}

In a systematic study of the bleaching behavior, we first investigated the dependence of the fit parameters $\tau_{f}$, $\tau_{s}, A_{f}$, and $A_{s}$ on the radiation exposure time $t_{e}$. As can be seen in Fig. 5(a), the relaxation times $\tau_{f}$ and $\tau_{s}$ remain constant within our accuracy when $t_{e}$ is varied by more than an order of magnitude from $t_{e}=20$ to $250 \mathrm{~s}$. The mean values we obtained were $\tau_{f}=18 \mathrm{~s}$ and $\tau_{s}=250 \mathrm{~s}$. The corresponding amplitudes $A_{f}$ and $A_{s}$ are shown in Fig. 5(b). Above $t_{e}=20 \mathrm{~s}$ the amplitude of the fast exponential $A_{f}$ has reached a nearly constant value, whereas $A_{s}$ still increases with UV exposure time; and, for the conditions used here, was found to saturate only for exposure times above $1200 \mathrm{~s}$.

\section{Ambient atmosphere}

It is well known that the photochromic behavior of $\mathrm{WO}_{3}$ is strongly influenced by the presence of reactive agents. Accordingly, it has been found that the photochromic response is increased considerably by the presence of hydrogenated vapor. ${ }^{14,15}$ This has been explained by the dissociation of hydrogen molecules on the surface and subsequent migration into the material. Furthermore, the bleaching behavior is strongly influenced by oxidizing agents like ozone ${ }^{3}$ or $\mathrm{H}_{2} \mathrm{O}_{2}$, as already mentioned in Sec. I.

In contrast, we have investigated the influence of inert gases on the photochromic effect. At first we irradiated a sample in nitrogen atmosphere at 1.5 bar $\left(t_{e}=20 \mathrm{~s}\right)$. Again, the ultraviolet light causes a strong increase of absorption, as shown in Fig. 6. After exposure, bleaching starts in a qualitatively similar way as it did in Fig. 4 for oxygen atmosphere. At long bleaching times, however, an important difference is observed: The sample now reaches a stable absorption level which is distinctly higher than the 


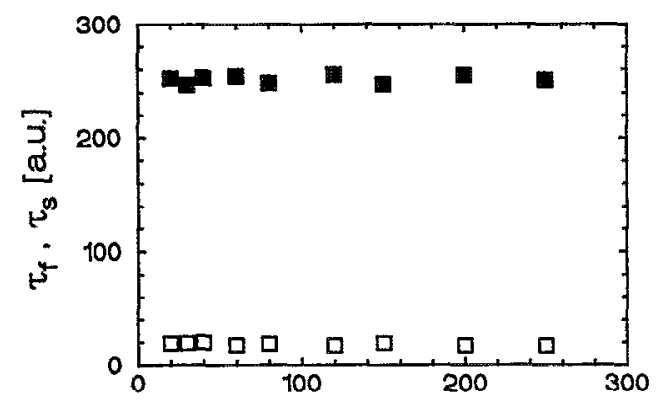

(a)

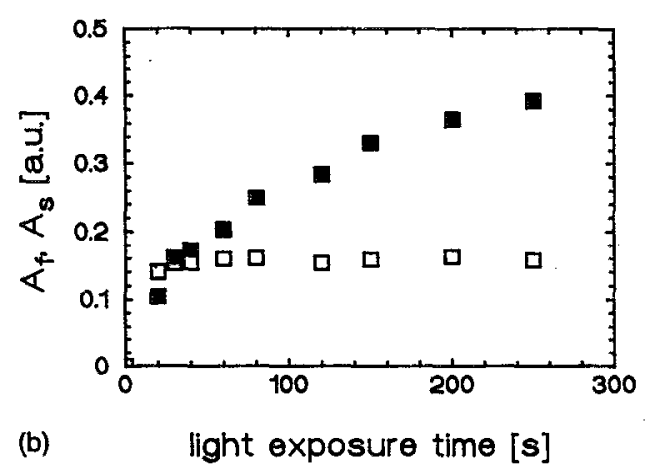

FIG. 5. Dependence of the fit parameters used in Eq. (1) on irradiation time $t_{e}$. Plotted are (a) relaxation times $\tau_{f}$ and $\tau_{s}$ and (b) the corresponding amplitudes $A_{f}$ and $A_{s}$. The fit parameters of the fast decay are marked by open squares. The filled squares correspond to the parameters of the slow decay.

value at the beginning of the measurement. This shows that the photochromic effect in a nitrogen atmosphere, in contrast to oxygen, is not completely reversible. Yet when a constant offset $A_{\infty}$ that describes the amount of irreversible absorption is subtracted, the data for the bleaching process can again be fitted by the sum of the two exponentials. The fit parameters are listed in Table I. Both relaxation times $\tau_{f}$ and $\tau_{s}$ are greater in nitrogen than in oxygen atmosphere, by $40 \%$ and $3 \%$, respectively. The amplitude $A_{f}$ is also increased in nitrogen (by nearly $20 \%$ ), whereas $A_{s}$ has

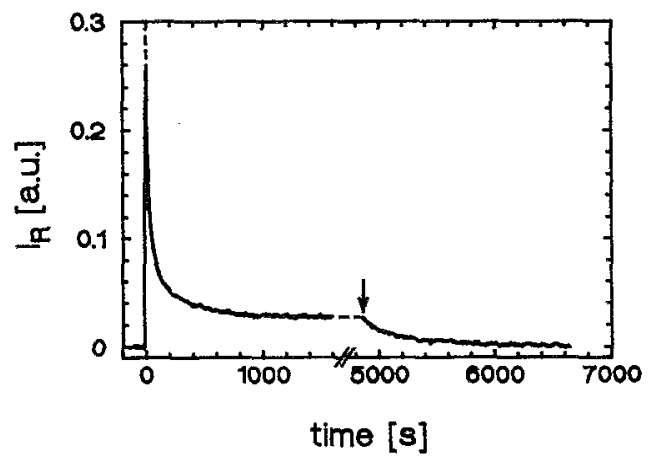

FIG. 6. Dynamics of $I_{R}$ during a coloration-bleaching experiment in nitrogen atmosphere. The arrow indicates the gas change from nitrogen to oxygen. The dashed line between $t=0$ and $t=1300 \mathrm{~s}$ is a fit (hardly visible because it is covered by the experimental curve) obtained by a double exponential, including an offset $A_{\infty}$.
TABLE I. Fit parameters for the bleaching behavior of a $10 \mathrm{~nm}$ tungsten oxide sample after UV irradiation for $20 \mathrm{~s}$ in different atmospheres. The parameters are obtained by fitting a double exponential decay to our data.

\begin{tabular}{llllll}
\hline \hline Atmosphere & $\begin{array}{c}A_{f} \\
\text { (a.u.) }\end{array}$ & $\begin{array}{c}A_{s} \\
\text { (a.u.) }\end{array}$ & $\begin{array}{c}A_{\infty} \\
(\text { a.u. })\end{array}$ & $\begin{array}{c}\tau_{f} \\
(\mathrm{~s})\end{array}$ & $\begin{array}{c}\tau_{s} \\
(\mathrm{~s})\end{array}$ \\
\hline Oxygen & 0.140 & 0.104 & 0 & 18.9 & 252 \\
Nitrogen & 0.169 & 0.053 & 0.032 & 26.3 & 260 \\
Helium & 0.166 & 0.060 & 0.032 & 26.4 & 247 \\
Argon & 0.169 & 0.059 & 0.030 & 26.4 & 250 \\
Vacuum & 0.162 & 0.055 & 0.034 & 26.9 & 253 \\
\hline
\end{tabular}

dropped by nearly a factor of 2 . The long-term absorption level $A_{\infty}$, which the sample had reached in $\mathrm{N}_{2}$ atmosphere after about $20 \mathrm{~min}$, was found to be constant at least on the scale of several hours.

Even in this state, however, the photochromic coloration process can still be made completely reversible by exchanging the gas atmosphere back to oxygen. This is also seen in Fig. 6, where the exchange from $\mathrm{N}_{2}$ to $\mathrm{O}_{2}$ is indicated by an arrow. The immediate onset of the bleaching process, as well as the relaxation back to the initial absorption level, is obvious. This behavior shows that there are at least two different decay channels for the color centers in $\mathrm{WO}_{3}$. Only one of them is affected by the ambient atmosphere, whereas the other is independent of it.

A closer look at Figs. 4 and 6 shows that for the same irradiation time $t_{e}$ the efficiency of the photochromic coloration in nitrogen is slightly enhanced compared to that in oxygen. This is expected, because during light exposure, the creation and decay of color centers occur simultaneously. Since the latter effect is partly suppressed in the case of a nitrogen atmosphere, the net coloration there should be larger, as it is indeed observed. Similar results to those in Fig. 6 are obtained if the samples are irradiated in helium, argon, or "vacuum" (meaning an ambient total pressure below $10^{-1} \mathrm{mbar}$ ). The fit parameters for these cases are also listed in Table I.

For nitrogen, helium, argon, and vacuum conditions the values found for the time constants as well as for the amplitudes were comparable. This means that in these cases both the sum $A_{f}+A_{s}$ (representing the reversible part of the coloration) and $A_{\infty}$ (the irreversible part) are essentially the same. The deviating results obtained for $\mathrm{O}_{2}$ atmosphere strongly suggest that oxygen plays an important role in the bleaching of photochromically colored tungsten oxide films.

To get more information about the origin of these two contributions-the reversible part and the irreversible part-we measured the relaxation behavior for different light exposure times of tungsten oxide under vacuum conditions. After each measurement, the sample chamber was filled with dry oxygen to force total bleaching of the sample. With this procedure we ascertained that all measurements started from the same absorption level. In order to check for correlations between the contributions Fig. 7 shows plots of the reversible part $A_{f}+A_{s}$ versus the irreversible part $A_{\infty}$. Up to irradiation times $t_{e} \approx 300 \mathrm{~s}$, corresponding in this plot to $A_{m}=2.4$, we found a roughly linear 


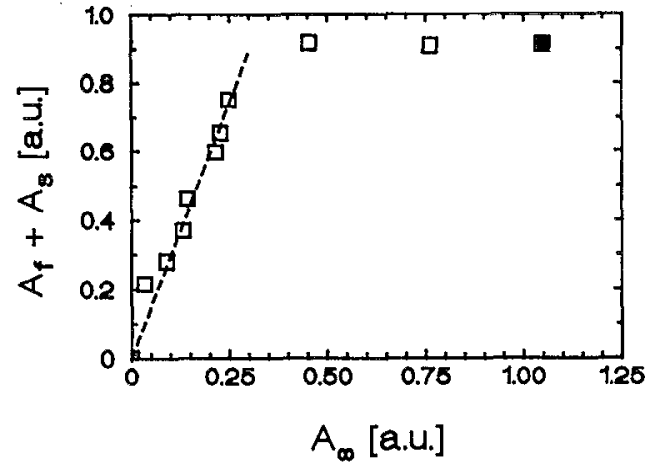

FIG. 7. Reversible $\left(A_{f}+A_{s}\right)$ and irreversible $\left(A_{\infty}\right)$ photochromic effect of a tungsten oxide layer illuminated in vacuum. The filled square corresponds to the maximum photochromic effect obtained with the same sample.

dependence between the reversible and the irreversible bleaching effect. Then, however, although the absorption of the sample can still be increased beyond this time, the reversible part saturates. Therefore we conclude that only a limited number of the total amount of color centers in a $\mathrm{WO}_{3}$ sample decays independently of the ambient atmosphere. We will discuss this point in some more detail next.

\section{Coloration process}

Up to now, we have dealt with the bleaching process that determines the absorption dynamics when the UV light source is turned off. Now we want to focus on the coloration process. To gain insight into the basic mechanisms that lead to the creation of color centers we investigated the coloration efficiency as a function of the wavelength $\lambda$ to which the sample was exposed. In order to avoid samples with the decay processes already taking place from irradiation and complicate our analysis, we have only used samples where bleaching is negligible. It has turned out in the course of our experiments that this is the case for $\mathrm{WO}_{3}$ films with a considerably larger thickness than before. As an example, Fig. 8 shows the behavior of a 340-nm-thick tungsten oxide layer irradiated by UV light in an oxygen atmosphere. The decay appears to be largely

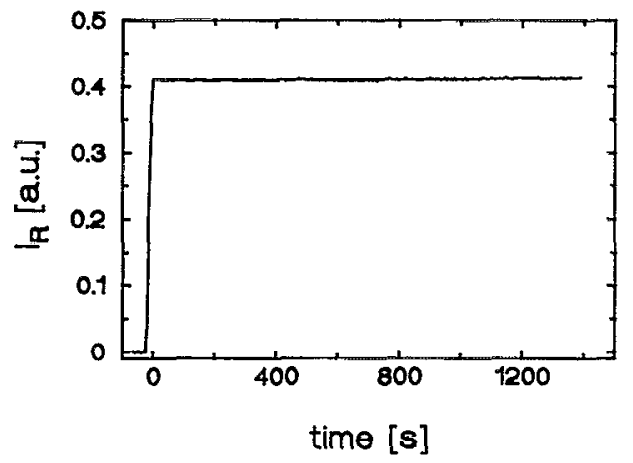

FIG. 8. $I_{R}$ as a function of time for a $340 \mathrm{~nm}, \mathrm{WO}_{3}$ sample irradiated in air. No relaxation is observed on the scale of several hours.

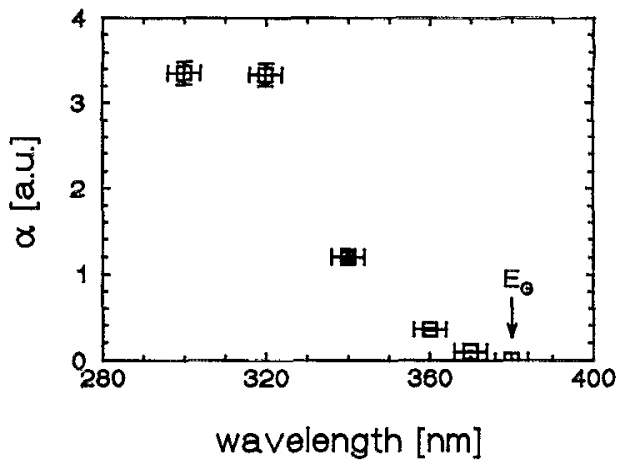

FIG. 9. Wavelength dependence of the coloration rate $\alpha$ of a $340 \mathrm{~nm}$ $\mathrm{WO}_{3}$ film. The onset at $380 \mathrm{~nm}$ is in agreement to the band gap energy of tungsten oxide (shown by the arrow).

suppressed. A possible explanation is that the interface between the tungsten oxide and the surrounding atmosphere plays a key role in the decay process.

Using such $340-\mathrm{nm}$-thick films, we have studied, the wavelength dependence of the coloration efficiency. The samples were irradiated with monochromatic UV light for several seconds, and the resulting linear increase of the absorption was recorded as a function of time. From the slope of the curves thus obtained, the coloration rate $\Delta \epsilon^{\prime \prime} / \Delta t$ was determined and was normalized to the incident light intensity $I_{\text {inc }}$. Results for these normalized coloration rates $\alpha$ are plotted in Fig. 9. For wavelengths down to $380 \mathrm{~nm}$, no photochromic effect could be detected; but, however, there is a strong increase of $\alpha$ towards shorter wavelengths. The onset of the photochromic effect is in good agreement with the band gap energy $E_{G}=3.25 \mathrm{eV}$ (indicated in Fig. 9 by an arrow) of thermally evaporated tungsten oxide films. ${ }^{3}$ This implies that in the wavelength range where electron-hole pairs are created inside the sample, color centers are also formed. Both processes are apparently closely connected to each other, as shown by the following comparison: The creation rate of electron-hole pairs $\eta$ by photons is known to depend strongly on the incident photon wavelength. Near the band edge one can write to good approximation ${ }^{16}$

$$
\eta h v \propto\left(h v-E_{G}\right)^{n / 2} .
$$

For crystalline samples the value of $n$ depends on whether the interband transition is direct $(n=1)$ or indirect ( $n$ $=4$ ). For amorphous tungsten oxide films the electron creation rate $\eta$ is known to obey Eq. (3) with $n=4 .{ }^{17}$ Now we want to compare this with our results obtained for the creation rate $\alpha$ of color centers. For this purpose wc have plotted in Fig. 10 the quantity $\alpha h v$ in analogy to Eq. (3) as a function of $\left(h v-E_{G}\right)$ in a double $\log$ plot. The slope of the solid line corresponds to $n=4$, which shows that the optical absorption rate $\alpha$ follows the same functional dependence on $\left(h v-E_{G}\right)$ as the electron hole-pair generation rate $\eta$. This result strongly suggests that coloration proceeds via the generation of charge carriers. 


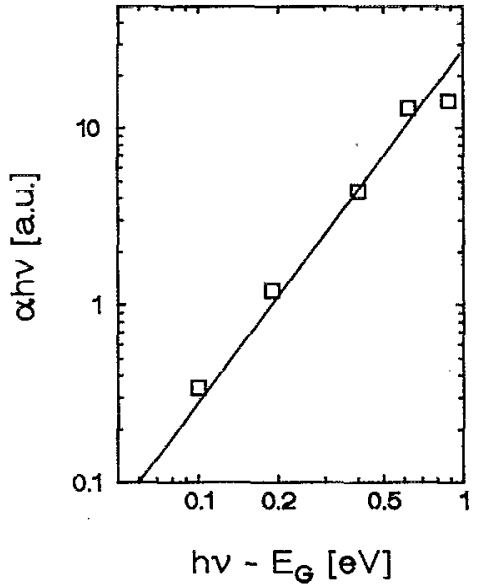

FIG. 10. Dependence of $(\alpha h v)$ for $\mathrm{WO}_{3}$ films on photon energy with respect to the band gap. The solid line corresponds to a slope of 2 , which is in agreement with the functional dependence found for the electronhole creation rate in tungsten oxide.

\section{DISCUSSION}

Evaporated tungsten oxide layers consist of a network of corner-sharing $\mathrm{WO}_{6}$ octahedron units, that are capable of forming clusters of different sizes. ${ }^{18}$ These clusters are considered to be connected to each other by $\mathrm{W}-\mathrm{O}-\mathrm{W}$ or hydrogen bonds, the latter by means of incorporated water. ${ }^{19}$ Electron spectroscopy for chemical analysis (ESCA) and RBS measurements determined the $\mathrm{H}_{2} \mathrm{O} / \mathrm{W}$ ratio for freshly evaporated films to about $0.15,{ }^{20}$ whereas storage in air can increase this value to $0.45 .{ }^{21}$ We will show that this incorporated water is probably the key to understanding our results of the photochromic effect of thin tungsten oxide films.

It was already demonstrated by Butler ${ }^{22,23}$ that a water-based electrolyte that is in contact with a single crystal of $\mathrm{WO}_{3}$ can be decomposed by irradiation with light into hydrogen and oxygen. This process is called photoelectrolysis and is well known for other semiconductors, too. ${ }^{24}$ The reaction is started by the generation of electronhole pairs by irradiating the sample with ultraviolet light $\left(E>E_{G}\right)$. The holes can weaken the $\mathrm{H}-\mathrm{O}$ bond of water molecules, leading to water decomposing into protons and highly reactive oxygen atoms. Thus one can write

$$
\mathrm{H}_{2} \mathrm{O}+2 h^{+} \stackrel{h v}{\rightleftharpoons} \mathrm{O}+2 \mathrm{H}^{+} \text {. }
$$

In order to prevent the back-reaction to occur, the particles have to be separated immediately. If, e.g., an external field is applied, the protons will drift to the cathode, where they recombine to hydrogen. In the case of crystalline $\mathrm{WO}_{3}$ this process will finally lead to the formation of $\mathrm{H}_{2}$ and $\frac{1}{2} \mathrm{O}_{2} \cdot{ }^{22,23}$

In the case of the photochromic effect, however, where no voltage is applied to the sample, the situation is somewhat different. If the nascent oxygen radicals could be bound in intermediate trapping states like oxygen vacancies, this would also avoid the back-reaction of Eq. (4). Then the excitation of electron-hole pairs in such a sample would give rise to the creation of metastable protons and electrons, which are capable of forming color centers. Indeed, evaporated tungsten oxide films contain a lot of oxygen vacancics which usually lead to nonstoichiometric $\mathrm{WO}_{3-x}$ films, where $x$ has been determined for thermally evaporated films to be about 0.15 . $^{20}$

Taking these oxygen vacancies into account, we suggest that the following reaction takes place in a tungsten oxide layer during UV exposure:

$$
\begin{aligned}
2 \mathrm{~W}^{+V I} \mathrm{O}_{3} \mathrm{H}_{2} \mathrm{O} & \stackrel{h v}{\rightleftharpoons} 2 \mathrm{~W}^{+V I} \mathrm{O}_{3}+2 \mathrm{H}^{+}+2 e^{-}+\mathrm{O} \\
& \rightleftharpoons 2 \mathrm{HW}^{+V} \mathrm{O}_{3}+\mathrm{O} .
\end{aligned}
$$

This equation describes the decomposition of water with optically excited electron-hole pairs and the subsequent transformation of tungsten oxide into the hydrogen tungsten bronze $\mathrm{H}_{x} \mathrm{~W}^{+V} \mathrm{O}_{3}$. It explains the experimentally observed proportionality between coloration due to the formation of $\mathrm{W}^{+V}$ centers and the number of generated electron-hole pairs (cf. Fig. 10). The oxygen vacancies in the layer are then essential for trapping the nascent oxygen generated, according to the right hand side of Eq. (5). Since the $\mathrm{WO}_{3}$ films are very thin, it is to be expected that part of the trapped oxygen will leave the sample during coloration. If $\mathrm{O}_{2}$ is added to the sample from outside after the irradiation, the loss of oxygen during coloration can be compensated for and a complete relaxation will occur, as we have demonstrated. If, on the other hand, the sample is in vacuum or is exposed to an atmosphere not containing $\mathrm{O}_{2}$, the oxygen which leaves the $\mathrm{WO}_{3}$ layer will be lost for the following bleaching process, and, therefore, only partial bleaching should occur. We ascribe this reversible contribution $A_{f}+A_{s}$ of the absorption to oxygen still trapped at vacancy sites after coloration, whereas $A_{\infty}$ is due to the amount of $\mathrm{O}_{2}$ that has left the sample.

For the case of complete coloration, we can get a rough estimate for the relationship between the irreversible and the reversible parts of bleaching. Assuming that the composition of our samples $\mathrm{WO}_{3-x} y \mathrm{H}_{2} \mathrm{O}$ is given by $x \approx 0.15$ and $y$ ranging between 0.15 and 0.45 (corresponding to an oxygen substoichiometry of $5 \%$ and a water content between $15 \%$ and $45 \%),{ }^{20,21}$ this means that the ratio of the maximum number of oxygen atoms created by the decomposition of water $(y)$ and the number of available oxygen vacancies $(x)$ ranges between 1 and 3, depending on the water content. Assuming, furthermore, that all the vacancies are occupied when the coloration is saturated, according to the above, we have $0 \leqslant A_{\infty} /\left(A_{f}+A_{s}\right) \leqslant 2$. Experimentally, we determined this value to be around 1 , as it can be seen from the filled square in Fig. 7, which corresponds to the maximum coloration of the investigated film. This is consistent with the proposed model.

It has been pointed out that the light-induced decomposition of water and, eventually, the creation of color centers require trapping states for the nascent oxygen atoms that are usually present in evaporated tungsten oxide films. If, however, no vacancies for oxygen exist in a $\mathrm{WO}_{3}$ sample at all, this should have drastic consequences for the photochromic effect. The generated oxygen cannot immediately be trapped, causing a rapid back-reaction into water and, 
hence, no coloration should occur. In fact, results of Gerard et al. confirmed this idea, since only a very weak photochromic effect is present in stoichiometric $\mathrm{WO}_{3}$ films. 25

Finally, we would like to address the possible relevance of the present results for the electrochromical coloration process. The nature of the color centers generated electrochemically and by $U V$ radiation is the same, namely, $\mathrm{W}^{+V}$ states, giving rise to an identical absorption band in the infrared region in both cases. ${ }^{6}$ We have shown that bleaching of photochromically, as well as electrochromically, generated color centers can be increased by exposing the samples to an oxidizing atmosphere or fluid $\left(\mathrm{H}_{2} \mathrm{O}_{2}\right)$, respectively. Therefore the question arises whether the electrochromic process can be explained by a mechanism similar to the one proposed here for the photochromic effect. RBS measurements confirmed that the hydrogen content of the samples is not changed during the electrochromic coloration. This strongly suggests that the hydrogen required for the formation of the hydrogen bronze $\mathrm{HWO}_{3}$ is alrcady present inside the sample, in contradiction to the double-charge-injection model. Further experiments are being prepared in order to determine whether the electrochemical coloration can also be understood in terms of water decomposition similar to the photochromic process studied here.

\section{CONCLUSIONS}

In summary, we have investigated the photochromic effect of thin evaporated tungsten oxide films by means of optically excited surface plasmons. For the first time, the dynamics of this process could be resolved on a time scale as short as seconds. In order to study the influence of the ambient atmosphere on the photochromic behavior, coloration-bleaching experiments were performed under $\mathrm{O}_{2}, \mathrm{~N}_{2}, \mathrm{He}, \mathrm{Ar}$, and vacuum conditions. The results clearly show that bleaching to the original absorption state only occurs if oxygen is offered to the sample from outside. Otherwise, the decay of color centers remains incomplete until the ambient atmosphere is changed to $\mathrm{O}_{2}$. Furthermore, we investigated the coloration behavior as a function of the incident light wavelength. The comparison of the results to the spectral dependence of the optically excited electron-hole pair creation rate indicates that there is a close relationship between both processes.

All these results can be understood in terms of a model based on the light-induced decomposition of water, which is incorporated in a considerable amount into tungsten oxide films. By means of incident UV light, electron-hole pairs are created and finally lead to the formation of pro- tons and electrons required for the formation of color centers. The probability of this reaction strongly depends on the amount of oxygen vacancies inside the sample, since during this process oxygen radicals are created and have to be trapped to avoid a rapid back-reaction. In accordance with these ideas, stoichiometric $\mathrm{WO}_{3}$ films show only a very weak photochromic effect.

The time dependence of the bleaching can be fitted very well to the sum of the two exponential decays whose physical interpretation is not yet clear. Attempts are in progress to develop a quantitative model for the dynamics of the photochromic effect.

\section{ACKNOWLEDGMENTS}

The authors wish to acknowledge helpful discussions with $\mathrm{K}$. Bange. This work was supported by Zentrum II für Energieforschung at the University of Konstanz.

${ }^{1} \mathrm{~K}$. Bange and T. Gambke, Adv. Mater. 1, 10 (1990).

${ }^{2}$ C. G. Granquist, Solid State Mater. Sci. 16, 291 (1990).

${ }^{3}$ S. K. Deb, Philos. Mag. 27, 801 (1973).

${ }^{4}$ A. Temmink, O. Anderson, K. Bange, H. Hantsche, and X. Yu, Thin Solid Films 192, 211 (1990).

${ }^{5}$ R. Gazzinelli and O. F. Schirmer, J. Phys. C 10, L145 (1977).

${ }^{6}$ A. I. Gavrilyuk, V. G. Prokhvatilov, and F. A. Chudnovskii, Sov. Phys. Solid State 24, 558 (1982).

${ }^{7}$ R. S. Crandall and B. W. Faughnan, Appl. Phys. Lett. 26, 120 (1975).

${ }^{8}$ W. Wagner, F. Rauch, C. Ottermann, and K. Bange, Nucl. Instr. Methods Phys. Res. B 50, 27 (1990).

${ }^{9}$ R. B. Goldner, T. E. Haas, G. Seward, K. K. Wong, P. Norton, G. Foley, G. Berera, G. Wei, S. Schulz, and R. Chapman, Solid State Ionics 28, 1715 (1988).

${ }^{10}$ Y. Shigesato, Jpn. J. Appl. Phys. 30, 1457 (1991).

${ }^{11}$ J. R. Sambles, G. W. Bradberry, and F. Yang, Contemp. Phys. 32, 173 (1991).

${ }^{12}$ R. Keller, Diploma thesis, University of Mainz, 1987.

${ }^{13}$ W. Siebrand and T. A. Wildman, Acc. Chem. Res. 19, 238 (1986).

${ }^{14}$ A. I. Gavrilyuk, T. G. Lanskaya, and F. A. Chudnovskii, Sov. Phys. Tech. Phys. 32, 964 (1987).

${ }^{15}$ M. Nagasu and N. Koshida, Appl. Phys. Lett. 57, 1324 (1990).

${ }^{16} \mathrm{E}$. J. Johnson, in Semiconductors and Semimetals, edited by R. K. Willardson and A. C. Beer (Academic, New York, 1967), Vol. 3, Chap. 6.

${ }^{17}$ M. Kitao, S. Yamada, S. Yoshida, H. Akram, and K. Urabe, Sol. Energy Mater. Sol. Cells 25, 241 (1992).

${ }^{18}$ II. R. Zeller and H. U. Beyeler, Appl. Phys. 13, 231 (1977).

${ }^{19}$ T. C. Arnoldussen, J. Electrochem. Soc. 128, 117 (1981).

${ }^{20}$ BmfFT Report: "Stabilisierung von oxidischen dünnen Schichten" (Stabilization of thin oxide films) BMFT-FKZ 13 N 5476 (1991)

${ }^{21} \mathrm{~F}$. Rauch, W. Wagner, and K. Bange, Nucl. Instrum. Methods B 42, 264 (1989).

${ }^{22}$ M. A. Butler, R. D. Nasby, and R. K. Quinn, Solid State Commun. 19, 1011 (1976).

${ }^{23}$ M. A. Butler, J. Appl. Phys. 48, 1914 (1977).

${ }^{24}$ J. F. Rabek, in Photochemistry and Photophysics, edited by J. F. Rabek (CRC, Boca Raton, FL, 1991), Vol. 3.

${ }^{25}$ P. Gerard, A. Deneuville, and R. Courths, Thin Solid Films 71, 221 (1980). 